\title{
Morfología polínica de las especies chaqueñas del género Schinopsis Engl. (Anacardiaceae) y sus implicancias taxonómicas
}

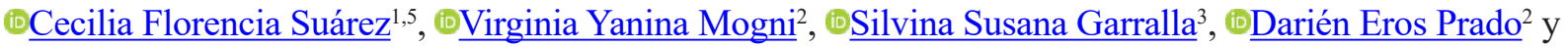 \\ - Olga Gladys Martínez ${ }^{1,4}$
}

Recibido: 14 agosto 2018; aceptado: 17 junho 2019

Como citar: Suárez, C.F., Mogni, V.Y., Garralla, S.S., Prado, D.E. \& Martínez, O.G. 2019. Morfología polínica de las especies chaqueñas del género Schinopsis Engl. (Anacardiaceae) y sus implicancias taxonómicas. Hoehnea 46: e762018. http://dx.doi.org/10.1590/2236-8906-76/2018.

RESUMEN - (Morfología polínica de las especies chaqueñas del género Schinopsis Engl. (Anacardiaceae) y sus implicancias taxonómicas). El género Schinopsis Engl. (Anacardiaceae) tiene ocho especies, de las cuales en el presente trabajo se describen palinológicamente a S. balansae Engl., S. boqueronensis Mogni \& Oakley, S. cornuta Loes., S. haenkeana Engl., S. heterophylla Ragonese \& J.A. Castigl. y S. lorentzii (Griseb.) Engl. Las muestras se tomaron de individuos en su ambiente natural y de ejemplares disecados. Los granos de polen fueron acetolizados y se estudiaron mediante microscopía de luz y electrónica de barrido. Estos son isopolares, radiosimétricos, con forma suboblato, esferoidal, prolato-esferoidal y prolato, de ámbito subcircular, subtriangular o triangular lobado; zonotremos, tricolporados y con endoaperturas lalongadas, de tamaño pequeño a mediano, con diámetro polar de 18-36 $\mu \mathrm{m}$ y diámetro ecuatorial de 16-28 $\mu \mathrm{m}$. La exina es semitectada, estriada a supraestriada-infrarreticulada. Esta caracterización palinológica aporta nueva información, la que en combinación con el estudio de otros caracteres, contribuye a la delimitación taxonómica de las especies chaqueñas de Schinopsis Engl., principalmente $S$. haenkeana de $S$. lorentzii.

Palabras-clave: Argentina, Bolivia, quebracho, Paraguay, polen

RESUMO - (Morfologia polínica das espécies chaquenhas do gênero Schinopsis Engl. (Anacardiaceae) e suas implicações taxonômicas). O gênero Schinopsis Engl. (Anacardiaceae) tem oito espécies, das quais, no presente trabalho descrevem-se palinologicamente, a S. balansae Engl., S. boqueronensis Mogni \& Oakley, S. cornuta Loes., S. haenkeana Engl., S. heterophylla Ragonese \& J.A. Castigl. e S. lorentzii (Griseb.) Engl. As amostras foram coletadas de indivíduos em seu ambiente natural e de exemplares dissecados. Os grãos de pólen foram acetolisados e estudados por microscopia eletrônica de varredura. Estes são isopolares, radiossimétricos, com forma suboblata, esferoidal, prolato-esferoidal e prolato, de âmbito pequeno a mediano, com diâmetro polar de 18-36 $\mu \mathrm{m}$ e um diâmetro equatorial de 16-28 $\mu \mathrm{m}$. A exina é semitectada, estriada a supraestriada-infrarreticulada. Essa caracterização palinológica fornece novas informações que, em combinação com o estudo de outros caracteres, contribui para a delimitação taxonômica das espécies chaquenhas de Schinopsis Engl., principalmente $S$. haenkeana de S. lorentzii.

Keywords: Argentina, Bolívia, quebracho, Paraguai, pólen

\section{Introducción}

El género Schinopsis Engl. (Anacardiaceae, Anacardioideae) (Judd et al. 1999, Pell 2004) comprende ocho especies de árboles (Meyer \& Barkley 1973, Mogni 2015), con distribución geográfica restringida a los Bosques Secos Estacionales Neotropicales (BSEN o SDTF, sensu Prado 2000) y

1. Universidad Nacional de Salta, Facultad de Ciencias Naturales, Av. Bolivia 5150, (4400) Salta, Argentina

2. Universidad Nacional de Rosario, Facultad de Ciencias Agrarias, Botánica, C.C. N ${ }^{\circ}$ 14, e Instituto de Investigaciones en Ciencias Agrarias, IICAR-CONICET, (2125) Zavalla, Argentina

3. Universidad Nacional del Nordeste, Departamento de Biología, UNNE, CECOAL-CONICET Ruta 5, km 2,5, Av. Libertad 5500, (3400) Corrientes, Argentina

4. Universidad Nacional de Salta, Facultad de Ciencias Naturales, Instituto de Bio y Geociencias, IBIGEO-CONICET, Av. Bolivia 5150, (4400) Salta, Argentina

5. Autor para correspondencia: suarezflorenciace@gmail.com 
a los subtropicales chaqueños de Sud América (Prado 1993a, b). Se trata de un género de gran importancia ecológica, cultural y socio-económica nacional e internacional. Sus especies tienen alto valor ecológico, ya que suelen ser dominantes o codominantes en los ambientes en los que habitan.

Son árboles de mediano a gran porte que, en ocasiones, pueden alcanzar los $25 \mathrm{~m}$ de altura y 1,5 m. de diámetro (Barberis et al. 2012). La copa puede variar ampliamente, desde triangular invertida u obcónica en algunas especies, hasta ampliamente semicircular/hemisférica en otras; la mayoría de los individuos presentan en la porción más baja de la copa y sobre el tronco, un conjunto de ramas comúnmente llamado 'pollera' (Meyer \& Barkley 1973, Barberis et al. 2012). La presencia de espinas de origen caulinar es frecuente en casi todas las especies del género; las hojas pueden ser simples (i.e. S. balansae Engl.), compuestas imparipinnadas (e.g. S. lorentzii (Griseb.) Engl.) o de ambos tipos sobre un mismo individuo (i.e. S. boqueronensis Mogni \& Oakley, S. cornuta Loes. y $S$. heterophylla Ragonese \& J.A. Castigl.). Las flores, dispuestas en tirsos o panículas, son unisexuales, pequeñas, pentámeras, amarillo verdosas a color crema. Las flores masculinas constan de cinco estambres con filamentos de forma tubular y anteras aovado-oblongas, dorsifijas, de aproximadamente igual longitud que los filamentos (Meyer \& Barkley 1973, Muñoz 1990), con dos tecas tetraesporangiadas de dehiscencia longitudinal; y ovario vestigial (Muñoz 1990). Las flores femeninas poseen estaminodios no funcionales muy pequeños, y presentan ovario súpero, tricarpelar, unilocular, generalmente con tres estilos laterales. El fruto es samaroideo, semileñoso y lustroso, rojizo a pardo, con una única semilla. Sobre la porción seminífera, la presencia de restos estigmáticos en forma de cicatrices o 'cuernitos' puede ser más o menos notoria y de valor taxonómico (Meyer \& Barkley 1973, Muñoz 1990).

Notablemente, varios de los taxones endémicos de la región biogeográfica chaqueña, presentan algunas confusiones y problemas taxonómicos, dada la variabilidad y similitud inter e intraespecíficas; por ejemplo, las dos especies más conocidas, $S$. balansae y S. lorentzii ('quebracho colorado chaqueño' y el 'quebracho colorado santiagueño'), aparentemente se hibridan en el sector geográfico de contacto de sus poblaciones (Ragonese \& Castiglioni 1947). En esa área aparecen ejemplares con caracteres intermedios entre ambas, conocidos como 'quebracho mestizo' (S. heterophylla). Meyer
\& Barkley (1973) sumergieron a este binomio en la sinonimia de $S$. lorentzii, al postular que estos individuos de características intermedias serían simplemente juveniles de dicha especie. Sin embargo, Anzótegui (1971), del Pero de Martínez (1972) y más recientemente Mogni (2015) encontraron caracteres intermedios para $S$. heterophylla con respecto a $S$. balansae y $S$. lorentzii, por lo que reconocen a $S$. heterophylla dentro del rango específico. Por otra parte, Cabrera (1938) postuló que S. marginata Engl., el 'horko quebracho', es en realidad sólo una variedad de $S$. lorentzii, y creó la combinación $S$. lorentzii var. marginata (Engl.) Cabrera. En este sentido, Flores et al. (2013) por medio de análisis multivariado de caracteres morfológicos, sinonimizaron a $S$. marginata bajo $S$. lorentzii. Posteriormente, otro estudio indica que $S$. lorentzii y $S$. marginata aparentemente serían dos entidades diferenciables morfológica y molecularmente (Mogni 2015), siendo el nombre correcto para ese último taxón S. haenkeana (Mogni et al., 2017).

$\mathrm{Si}$ bien varios caracteres vegetativos foliares como el tipo de hoja, tamaño, textura, simetría, forma y aspecto del ápice y base, así como la serosidad y pilosidad de las hojas fueron considerados de importancia taxonómica (Ragonese \& Castiglioni 1947, Barkley 1962, Mogni et al. 2014, Mogni 2015), poco se conoce hasta el momento sobre la utilidad de los aspectos anatómicos y/o palinológicos como aporte a la diferenciación a nivel específico. En particular, el polen de Schinopsis ha sido escasamente estudiado; Anzótegui (1971) describió los granos de polen de tres especies de Schinopsis del nordeste argentino (S. balansae, S. heterophylla y S. quebrachocolorado $(=S$. lorentzii)), indicando a la escultura como reticulada a supraestriada-infrarreticulada. Luego el mismo autor, Anzótegui (2001), incluye a S. balansae en el Tipo Schinopsis balansae, Subtipo Lithraea molleoides por las características observadas con microscopio de luz y microscopio electrónico de barrido.

En relación a la complejidad observada a nivel morfológico para el estudio y la diferenciación de las especies chaqueñas del género, este trabajo tiene como objetivo aportar datos de la morfología del polen de las seis especies de Schinopsis restringidas a los bosques subtropicales chaqueños sudamericanos: S. balansae, S. boqueronensis, S. cornuta, S. heterophylla, $S$. lorentzii y $S$. haenkeana, con la finalidad de contribuir a la delimitación y caracterización de esos taxones. 


\section{Materiales y métodos}

E1 material estudiado proviene de flores de especímenes colectados en el ambiente natural, cuyos ejemplares de referencia fueron depositados en el Herbario UNR "Juan Pablo Lewis"; también se consideraron otros ejemplares disponibles en los herbarios CTES, FCQ, MCNS y UNR (Thiers 2015).

\section{Schinopsis balansae}

Material examinado: ARGENTINA. CoRRIENTES: Ruta Nac. 5, Banquina derecha, sentido NO-SE, 26-II-2011, V. Mogni et al. 54 (UNR). BRASIL. Mato Grosso Do Sul: Porto Murtinho, Hotel dos Camalotes, 18-II-1989, A. Pott. et al. 4613 (UNR). PARAGUAY. Ruta Trans Chaco, km 85, cerca de Benjamín Acebal, 16-XII-2011, M. Vera Jiménez et al. 3257 (FCQ).

\section{Schinopsis boqueronensis}

Material examinado: PARAGUAY. Ruta Trans Chaco entre Pioneros y 25 Leguas, a la derecha, dirección O-E, 16-XI-2011, M. Vera Jiménez et al. 4240 (FCQ).

\section{Schinopsis cornuta}

Material examinado: BOLIVIA. Comunidad "El Vinal", Camino El Salvador-Carandaití, 29-X-1992, C. Saravia Toledo \& J. Nelson 11083 (CTES). PARAGUAY. Campo Aroma km 10, 22-X-1992, F. Mereles \& R. Degen 4838 (CTES).

\section{Schinopsis haenkeana}

Material examinado: ARGENTINA. JusuY: Dpto. El Carmen, Distrito Puesto Viejo, Loc. Puesto Viejo, Finca Cabeza de Toba o Cañadas, $10 \mathrm{~km}$ al $\mathrm{W}$ de R. N. 34, 968 m, 9-II-2006, G. Guzmán \& G. Villagra s.n. (MCNS 2101); Dpto. San Pedro, Arroyo del Medio, 15-II-1998, V. Mogni et al. 21 (MCNS). SALTA: Dpto. Guachipas, El Cebilar, N 2538,6' W $65^{\circ} 28,5^{\prime}, 1587$ m, 18-I-2011, O. Martínez \& D. Prado 2080 (MCNS); Dpto. Metán, Sierras de Metán, Finca Cachari, alrededores del campamento León, serranías $20 \mathrm{~km}$ al W de Juramento ladera N y NNE de Cerro el Plomo, 13-14-XI-2007, J. Tolaba 4331 (MCNS). SAN JUAN: Ruta Nac. 510, entrando por camino lateral, próximo a Las Tumanas, Propiedad privada Lucero, 27-V-2013, V. Mogni et al. 131 (UNR).

\section{Schinopsis heterophylla}

Material examinado: ARGENTINA. ChACo: Ruta Nac. 95, entre Cruce y Tres Isletas, 28-II-2011, V.
Mogni et al. 70 (UNR). Formosa: Ruta Nac. 95, km 25, 27-II-2011, V. Mogni et al.63. (UNR).

\section{Schinopsis lorentzii}

Material examinado: ARGENTINA. FormosA: Ruta Nac. 95, a pocos km del cruce con Ruta Nac. 81, 28-II-2011, V. Mogni et al. 72 (UNR); Ruta Nac. 27 casi llegando a la Ruta Nac. 95, 28-II-2011, V. Mogni et al. 74 (UNR).

De cada especie se analizaron entre 60-80 granos de polen. Los granos fueron acetolizados según la técnica convencional (Erdtman 1969), montados y sellados con gelatina glicerinada. Las descripciones de los granos comprenden: ámbito, endoaperturas (forma y tamaño de los ora), colpos, diámetro ecuatorial (E), diámetro polar $(\mathrm{P})$, forma determinada a partir de la relación P/E, ornamentación de la exina e Índice de Área Polar (IAP) según las propuestas de Erdtman (1952), Fernández \& Diez (1990), Sáenz Laín (2004) y Punt et al. (2007). Las medidas de E y P se expresan en valores promedios, entre paréntesis el menor y mayor valor registrado (tabla 1).

Las observaciones y mediciones se realizaron con microscopio de luz (MO) Leica DM500 con cámara integrada, y para las observaciones con microscopía electrónica de barrido (MEB), los granos fueron montados sobre tacos, cubiertos con oro-paladio y analizados con Microscopio Electrónico de Barrido JEOL JSM 6480 LV SEM perteneciente al Laboratorio de Microscopía Electrónica de Barrido y Microanálisis de la Universidad Nacional de Salta.

\section{Resultados}

Las descripciones palinológicas de las especies estudiadas aquí son las siguientes:

Descripciones palinológicas

Características generales

El polen de las especies de Schinopsis: S. balansae, S. boqueronensis, S. cornuta, S. haenkeana, S. heterophylla y $S$. lorentzii, se caracterizan por ser radiosimétricos, isopolar, de ámbito subcircular, circular a subtriangular. Suboblato, esferoidal, prolatoesferoidal y prolato con $\mathrm{P}=18-36 \mu \mathrm{m}$ y $\mathrm{E}=16-28 \mu \mathrm{m}$. Tricolporados, colpos largos y angostos con membrana apertural granulada, de $19,6-23 \times 1,5-2,2 \mu \mathrm{m}$ y endoaperturas lalongadas de 1,7-3,2 × 2,6-3,7 $\mu \mathrm{m}$. Exina supraestriada-infrarreticulada, relación sexina/ nexina $=0,7-1,5 / 0,5-1 \mu \mathrm{m}$. 
Tabla 1. Mediciones palinológicas para las especies de Schinopsis. Forma, P (diámetro polar), E (diámetro ecuatorial), Sexina/Nexina, Endoapertura (largo $\times$ ancho), Colpo (largo $\times$ ancho), Índice de Área Polar (IAP).

Table 1. Palynological measurements for the Schinopsis species. Shape, P (polar diameter), E (equatorial diameter), Sexine/ Nexine, Endoaperture (length $\times$ width), Colpus (length $\times$ width), Polar Area Index (IAP).

\begin{tabular}{|c|c|c|c|c|c|c|c|}
\hline Especie & Forma & $\mathrm{P}(\mu \mathrm{m})$ & $\mathrm{E}(\mu \mathrm{m})$ & $\begin{array}{l}\text { Sexina/Nexina } \\
(\mu \mathrm{m})\end{array}$ & $\begin{array}{c}\text { Endoapertura } \\
\qquad(\mu \mathrm{m})\end{array}$ & Colpo $(\mu \mathrm{m})$ & $\begin{array}{l}\text { IAP } \\
(\mu \mathrm{m})\end{array}$ \\
\hline S. balansae & Oblato-esferoidal & $25(23-29)$ & $24(23-25)$ & $1 / 1$ & $3 \times 3,7$ & $23 \times 1,6$ & 0,2 \\
\hline S. boqueronensis & Prolato & $24(18-29)$ & $20(16-24)$ & $1 / 1$ & $1,7 \times 2,6$ & $19,6 \times 1,5$ & 0,2 \\
\hline S. cornuta & Prolato-esferoidal & $25(20-27)$ & $23(19-26)$ & $1 / 0.7$ & $2 \times 2,6$ & $19,6 \times 1,5$ & 0,2 \\
\hline S. haenkeana & Suboblato & $33(29-36)$ & $25(20-28)$ & $1 / 1$ & $2,5 \times 3$ & $22 \times 2$ & 0,3 \\
\hline S. heterophylla & $\begin{array}{l}\text { Suboblato a } \\
\text { esferoidal }\end{array}$ & $22(19-30)$ & $22(19-24)$ & $0.7 / 0.7$ & $2,5 \times 3$ & $20 \times 1,8$ & 0,4 \\
\hline S. lorentzii & Prolato-esferoidal & $27(26-29)$ & $26(24-28)$ & $1,5 / 0.5$ & $3,2 \times 3,6$ & $19,6 \times 2,2$ & 0,2 \\
\hline
\end{tabular}

Las mediciones palinológicas comparativas entre las especies de Schinopsis, se consignan en la tabla 1.

\section{Schinopsis balansae Engl.}

Figuras $1 \mathrm{a}-\mathrm{b}, 3 \mathrm{a}-\mathrm{b}$

Granos prolato-esferoidales. Ámbito circular a subcircular. Tricolporados. Colpos de $\pm 23 \times 1,6 \mu \mathrm{m}$. Endoapertura lalongada. Apocolpio de ca. $5 \mu \mathrm{m}$. Exina supraestriada-infrarreticulada de ca. $2 \mu \mathrm{m}$ de espesor, sexina y nexina de aproximadamente igual espesor.

MEB: confirma que la exina es supraestriadainfrarreticulada en los mesocolpios y estriada en las áreas polares; estrías ramificadas y subparalelas de ca. 0,6 $\mu \mathrm{m}$ de ancho, lúmenes del infrarretículo irregulares, de formas y tamaños variados. Gránulos de la membrana apertural densamente distribuidos de $\geq 0,4 \mu \mathrm{m}$ de diám.

Observaciones: plantas únicamente con hojas simples. Esta especie crece principalmente en Argentina y Paraguay, pero también se encuentra en una reducida área de Brasil y Bolivia. Se distribuye en la región chaqueña húmeda.

\section{Schinopsis boqueronensis Mogni \& Oakley}

Figuras $1 \mathrm{c}-\mathrm{d}, 3 \mathrm{c}-\mathrm{d}$

Granos prolatos, raramente subprolatos. Ámbito subcircular. Tricolporados. Colpos de $\pm 19,6 \times 1,5 \mu \mathrm{m}$. Endoapertura lalongada. Apocolpio de ca. 4,7 $\mu \mathrm{m}$. Exina supraestriada-infrarreticulada de ca. $2 \mu \mathrm{m}$ de espesor; sexina y nexina de aproximadamente igual espesor.

MEB: Confirma que la exina es supraestriadainfrarreticulada; estrías largas (algunas de polo a polo), ramificadas, ligeramente sinuosas y paralelas de ca. 0,4 $\mu \mathrm{m}$ ancho. Lúmenes del infraretículo irregulares, de formas y tamaños variados. Gránulos de la membrana apertural de 0,2-07 $\mu \mathrm{m}$ de diám. densamente distribuidos.

Observaciones: plantas con hojas simples y compuestas pinnadas sobre un mismo ejemplar. S. boqueronensis presenta una distribución restringida al Chaco Seco en el centro-oeste de Paraguay (Presidente Hayes, Boquerón y Alto Paraguay) y en el este de Bolivia, fundamentalmente en el departamento de Santa Cruz.

\section{Schinopsis cornuta Loes.}

Figuras 1 e-f, 3 e-f

Granos prolatos esferoidales. Ámbito subtriangular. Tricolporados. Colpos de $\pm 19,6 \times 1,5 \mu \mathrm{m}$. Endoapertura lalongada. Apocolpio de ca. 6,5 $\mu \mathrm{m}$. Exina supraestriada-infrarreticulada de ca. $2 \mu \mathrm{m}$ de espesor; la sexina se presenta ligeramente de mayor espesor que la nexina.

MEB: Confirma que la exina es supraestriadainfrarreticulada; estrías cortas y sinuosas de ca. 2,5-4 ×0,5 $\mu \mathrm{m}$, lúmenes del infraretículo irregulares, de variadas formas y tamaños. Gránulos de la membrana apertural de $\leq 0,5 \mu \mathrm{m}$ de diám.

Observaciones: plantas con hojas simples y compuestas pinnadas sobre un mismo ejemplar. S. cornuta presenta una distribución muy semejante a $S$. boqueronensis, restringida al sector chaqueño en el centro-oeste de Paraguay y en el este de Bolivia, aunque algunos individuos eventualmente habitan áreas transicionales entre la región fitogeográfica Chaqueña y sabanas con elementos del Cerrado (Mogni et al. 2014). 


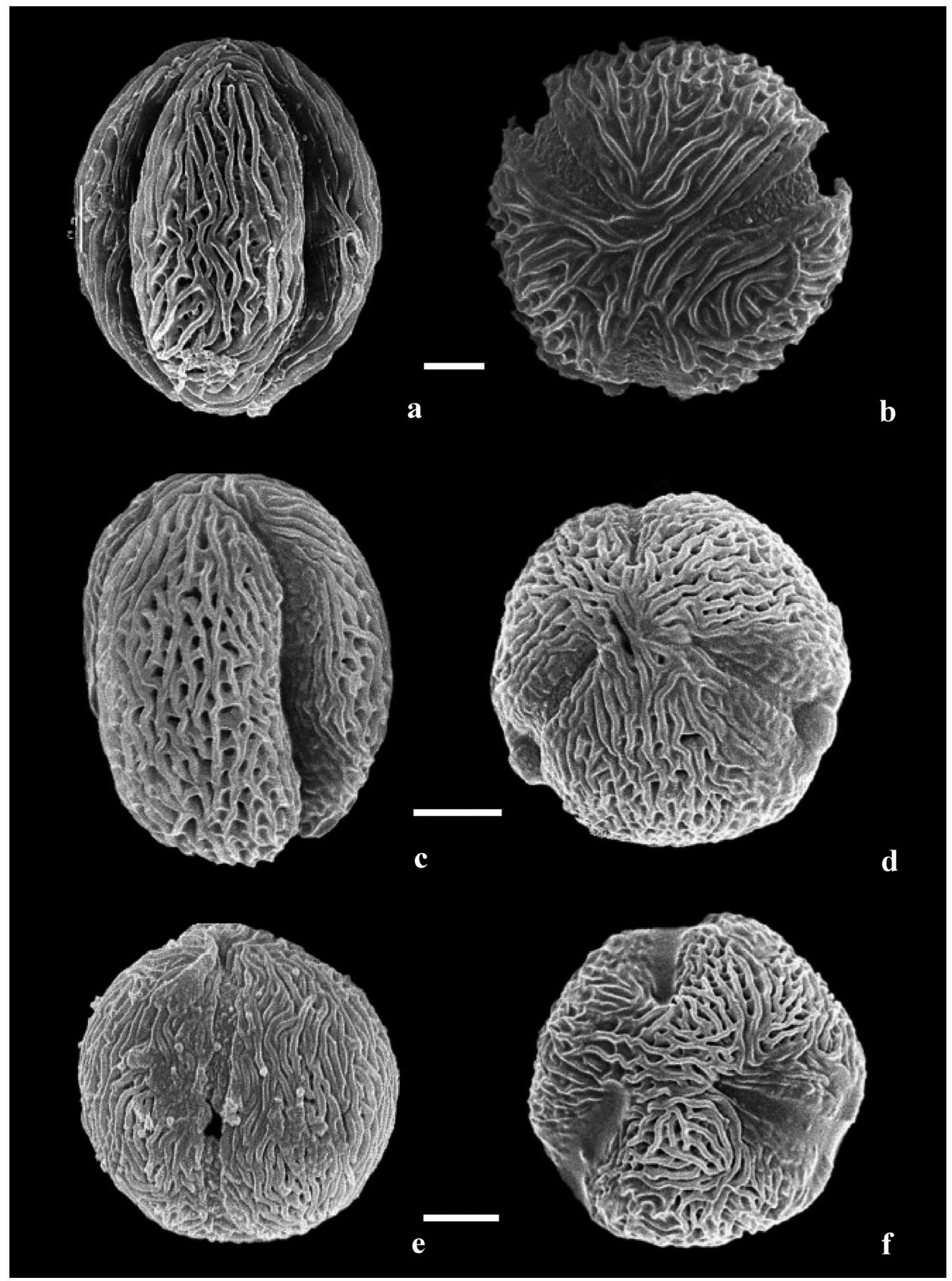

Figura 1.a-f. Fotomicrografías de MEB de los granos de polen de Schinopsis. a-b. S. balansae. a. Vista ecuatorial. b. Vista polar. c-d. S. boqueronensis. c. Vista ecuatorial. d. Vista polar. e-f. S. cornuta. e. Vista ecuatorial, detalle de la exoapertura con membrana apertural granular. f. Vista polar. Escala de barras $=5 \mu \mathrm{m}$.

Figure 1.a-f. SEM micrographs of Schinopsis pollen grains. a-b. S. balansae. a. Equatorial view. b. Polar view. c-d. S. boqueronensis. c. Equatorial view. d. Polar view. e-f. S. cornuta. e. Equatorial view, detail of ectoaperture with granular apertural membrane. f. Polar view. Scale bars $=5 \mu \mathrm{m}$. 


\section{Schinopsis haenkeana Engl.}

Figuras 2 a-b, 3 g-h

Granos suboblatos. Ámbito subcircular. Tricolporados. Colpos de $\pm 22 \times 2 \mu \mathrm{m}$. Endoapertura lalongada. Apocolpio de ca. $7 \mu \mathrm{m}$. Exina de ca. $2 \mu \mathrm{m}$ de espesor; nexina de igual espesor que la sexina.

MEB: confirma que la exina es supraestriadainfrarreticulada. Estrías paralelas y ramificadas de ca. 0,6 $\mu \mathrm{m}$ de ancho. Lúmenes del infrarretículo irregulares de diferentes formas y tamaños. Gránulos de la membrana apertural de ca. 0,2 $\mu \mathrm{m}$ de diám.

Observaciones: plantas únicamente con hojas compuestas pinnadas. En Argentina se encuentra en el sector centro a noroeste, desde San Juan hasta Jujuy. En Bolivia se lo encuentra en los departamentos de Tarija, Chuquisaca, Santa Cruz, Cochabamba y La Paz. Habita principalmente en el Chaco Serrano, aunque algunos individuos ingresan a la Selva Pedemontana lindera.

5. Schinopsis heterophylla Ragonese \& J.A. Castigl. Figuras $2 \mathrm{c}-\mathrm{d}, 3 \mathrm{i}-\mathrm{j}$

Granos suboblatos a esferoidales. Ámbito circular a subcircular. Tricolporados. Colpos de $\pm 20 \times 1,8 \mu \mathrm{m}$. Endoapertura lalongada. Apocolpio de ca. 4-5,5 $\mu \mathrm{m}$. Exina de 1,4-2 $\mu \mathrm{m}$ de espesor; nexina de igual espesor que la sexina.

MEB: confirma que la exina es supraestriadainfrarreticulada. Los muros están dispuestos muy juntos unos de otros, predominan los paralelos, levemente sinuosos y algunos bifurcados en sentido del eje polar, de 0,3-0,7 ×0,4 $\mu \mathrm{m}$. También se observan algunos muros transversales formando un infra retículo. Dado que los muros orientados en sentido del eje polar se destacan más que los transversales, dan al grano un aspecto de exina estriada. Gránulos de la membrana apertural de ca. 0,2 $\mu \mathrm{m}$ de diám.

Observaciones: plantas con hojas simples y compuestas pinnadas sobre un mismo ejemplar. En Argentina la especie se distribuye principalmente en forma de faja meridional atravesando el centro de Chaco y Formosa, una pequeña área del noroeste de Santa Fe y este de Santiago del Estero, donde confluyen las regiones chaqueñas húmeda y seca. Se encuentra a lo largo de gran parte del área de convivencia de S. lorentzii y $S$. balansae.

6. Schinopsis lorentzii (Griseb.) Engl.

Figuras 2 f-g. 3 k-1
Granos prolato-esferoidales. Ámbito subcircular. Tricolporados. Colpos de $\pm 19,6 \times 2,2 \mu \mathrm{m}$. Endoapertura lalongada. Exina de ca. $2 \mu \mathrm{m}$ de espesor; nexina de menor espesor que la sexina.

MEB: confirma que la exina es supraestriadainfrarreticulada. Estrías ramificadas y algunas sinuosas de ca. 0,5 $\mu \mathrm{m}$ de ancho, dispuestas a veces muy juntas. Lúmenes del infrarretículo irregulares de diferentes formas. Gránulos de la membrana apertural densamente distribuidos de ca. 0,2 $\mu \mathrm{m}$ de diám.

Observaciones: plantas únicamente con hojas compuestas pinnadas. Esta especie se encuentra en el centro-norte de Argentina, en el sur-este de Bolivia y en el sector oeste de Paraguay, principalmente en el Chaco Seco.

\section{Discusión}

Algunas características, tales como la forma de los granos de polen, el tipo de apertura y la escultura, están incluidas en la descripción presentada por Anzótegui (1971) para 12 especies de Anacardiaceae que crecen en el nordeste argentino, entre ellas: Schinopsis balansae, S. heterophylla y S. quebracho-colorado $(=S$. lorentzii). En relación a las dimensiones, se encuentra que los tamaños de los granos de polen de los ejemplares de $S$. balansae analizados en este estudio son semejantes a los citados por Anzótegui (1971) para la región nordeste de Argentina, pero levemente menores a los descritos para la misma región por Anzótegui (2001), quien indica valores de $\mathrm{P}=28,7(27-30) \mu \mathrm{m}$ y $\mathrm{E}=27,5(26-28) \mu \mathrm{m}$. En $S$. heterophylla y $S$. lorentzii las dimensiones registradas de P y E son semejantes, aunque con colpos levemente mayores a los mencionados $(1,5 \mu \mathrm{m})$ por Anzótegui (1971). Entre las seis especies estudiadas, S. lorentzii presenta mayores valores en el diámetro ecuatorial (E), sexina, longitud de la endoapertura y ancho de los colpos, S. boqueronensis registra el mayor diámetro polar (P) y S. heterophylla mayor índice de área polar (IAP) (Ver tabla 1).

El análisis del polen con MEB permitió corroborar que la exina es predominantemente supraestriadainfrarreticulada. Este tipo de exina también fue descrita para otras especies de Anacardiaceae, como Astronium balansae Engl., A. urundeuva Engl. y Schinus molle L. por Markgraf \& D’Antoni (1978), Athavale Pradeep (2014) describe a Anacardium occidentale L.como tipo estriado-reticulado y Anzotegui (2001) a Astronium balansae Engl., Litraea 


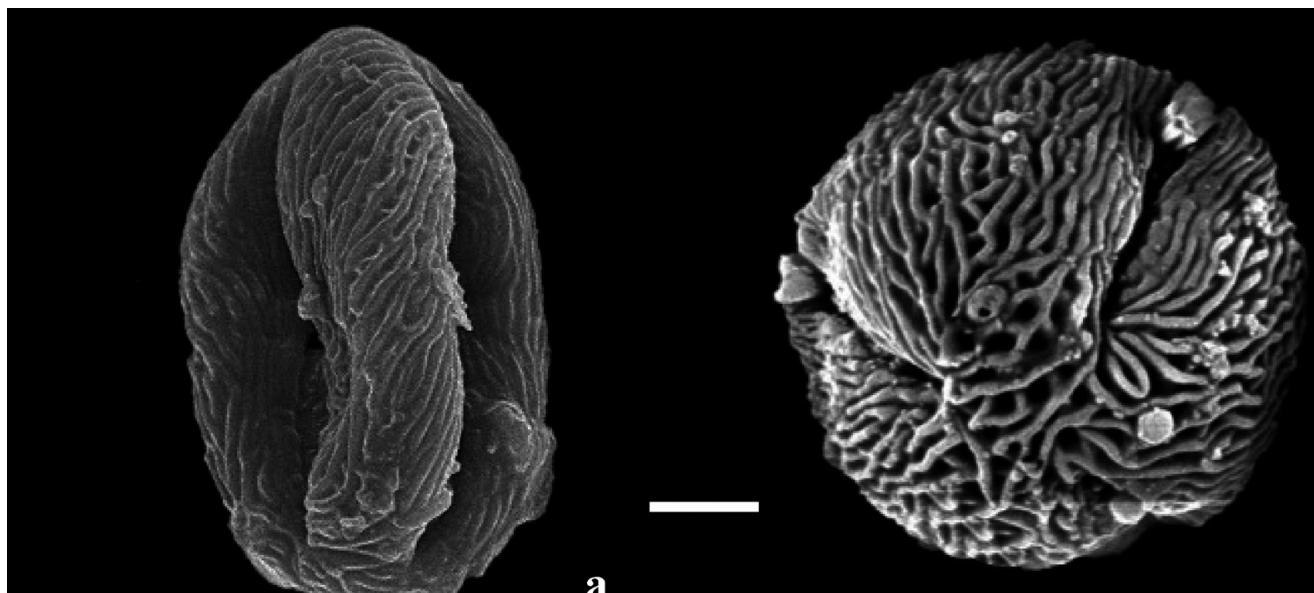

b

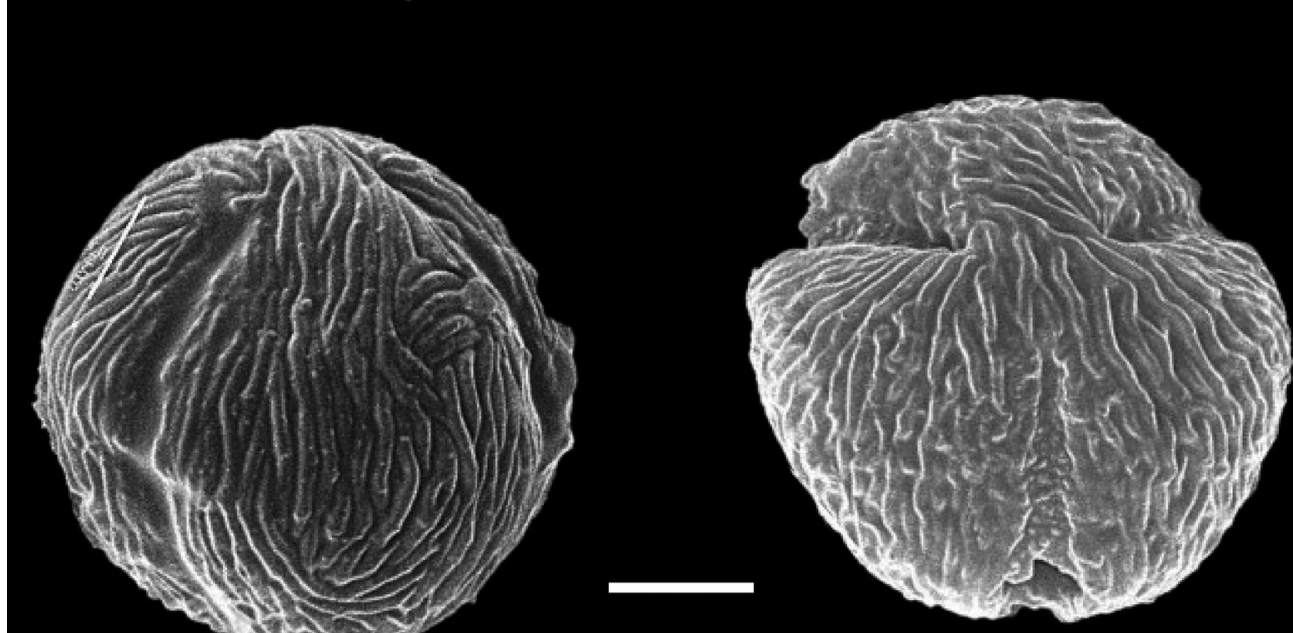

c

d
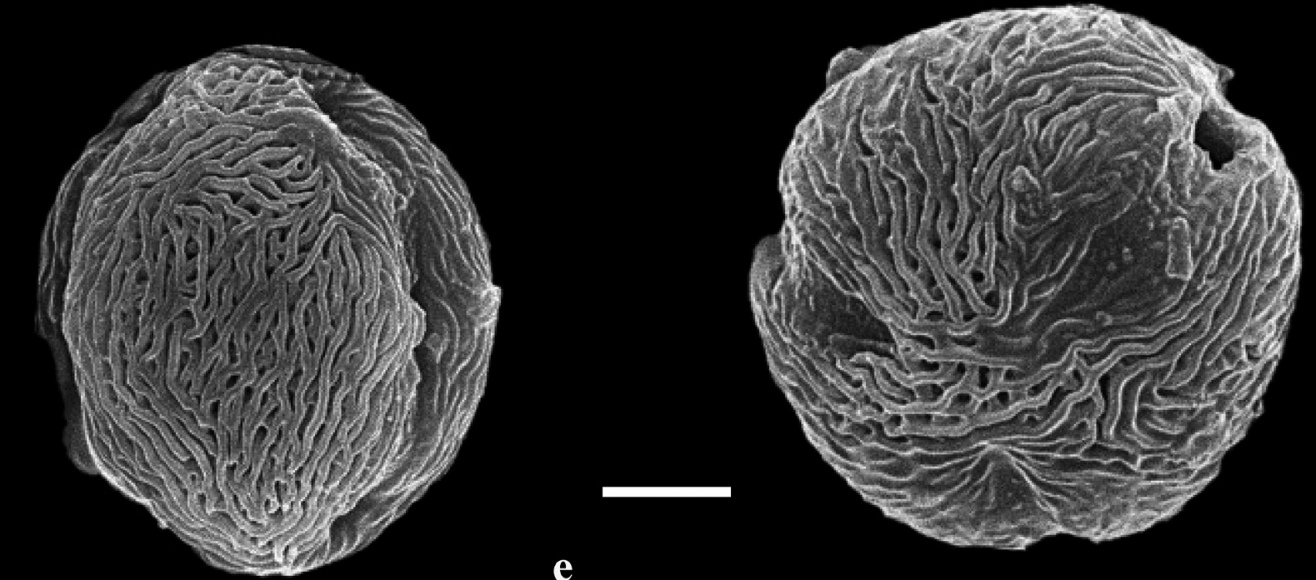

$\mathbf{e}$

Figura 2.a-f. Fotomicrografías de MEB de los granos de polen de Schinopsis. a-b. S. haenkeana. a. Vista ecuatorial. b. Vista polar. c-d. S. heterophylla. c. Vista ecuatorial. d. Vista polar, detalle de la membrana apertural. e-f. S. lorentzii. e. Vista ecuatorial. f. Vista polar, detalle de la exoapertura. Escala de barras $=5 \mu \mathrm{m}$.

Figure 2.a-f. SEM micrographs of Schinopsis pollen grains. a-b. S. haenkeana. a. Equatorial view. b. Polar view. c-d. S. heterophylla c. Equatorial view. d. Polar view, detail of apertural membrane. e-f. S. lorentzii. e. Equatorial view. f. Polar view, detail of apertural membrane. Scale bars $=5 \mu \mathrm{m}$. 


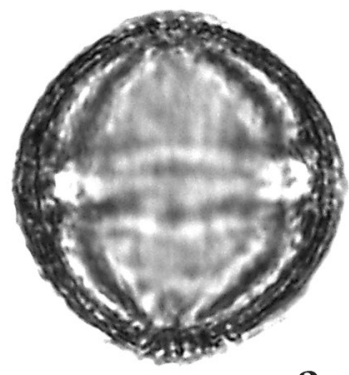

a

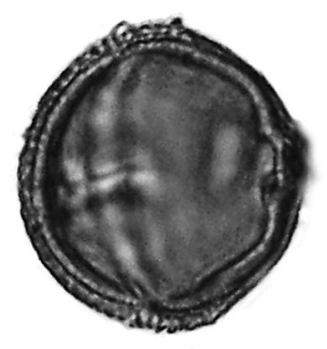

e

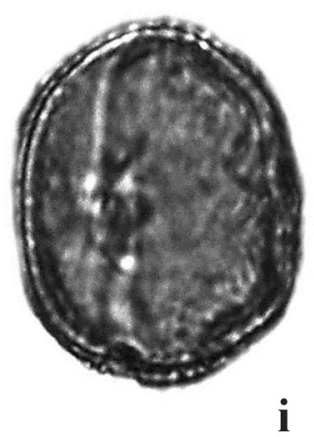

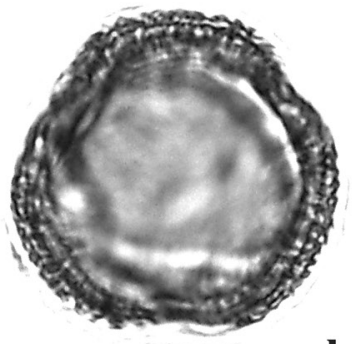

b

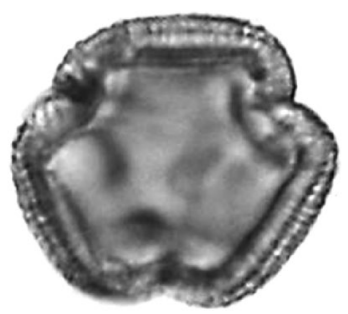

f

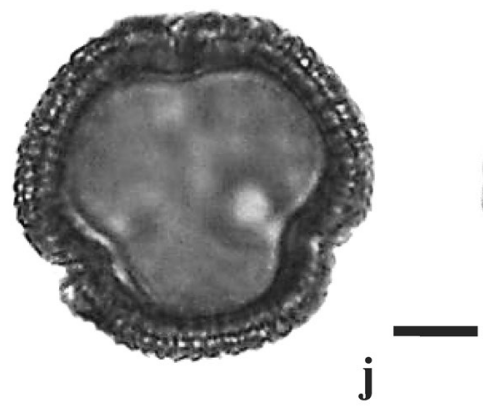

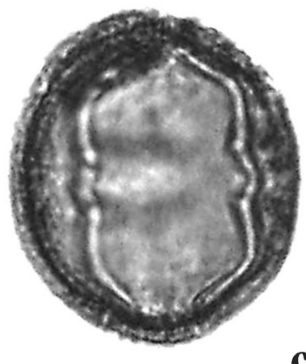

c

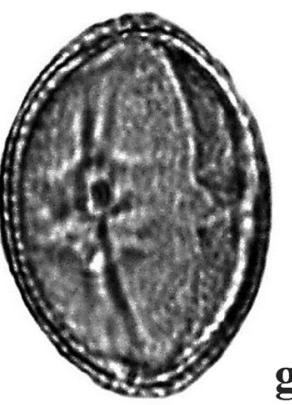

g

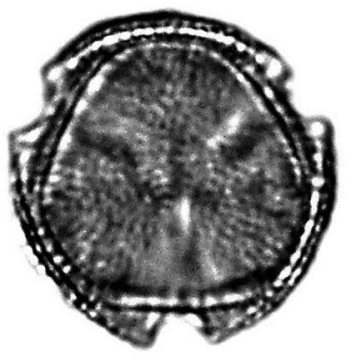

h

d
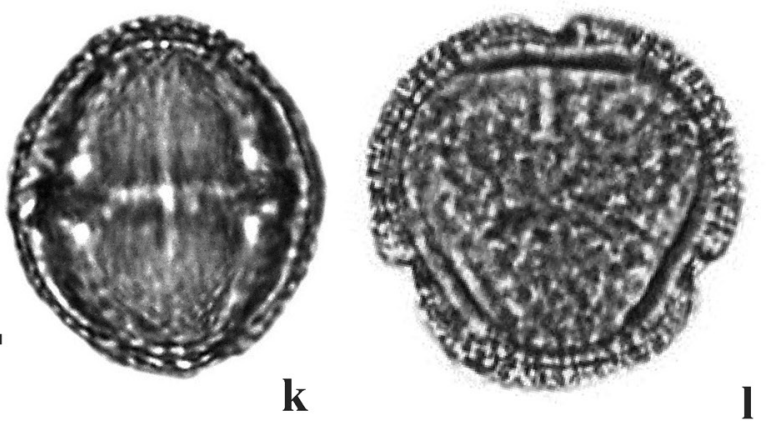

Figura 3.a-1. Fotomicrografias de los granos de polen de Schinopsis. a-b. S. balansae. a. Vista ecuatorial. b. Vista polar. c-d. S. boqueronensis. c. Vista ecuatorial. d. Vista polar. e-f. S. cornuta. e. Vista ecuatorial. f. Vista polar. g-h. S. haenkeana. g. Vista ecuatorial. h. Vista polar. i-j. S. heterophylla. i. Vista ecuatorial. j. Vista polar. k-1. S. lorentzii. k. Vista ecuatorial. 1. Vista polar. Escala de barra $=5 \mu \mathrm{m}$.

Figure 3.a-1. Light photomicrograph of Schinopsis pollen grains. a-b. S. balansae. a. Equatorial view. b. Polar view. c-d. S. boqueronensis. c. Equatorial view. d. Polar view. e-f. S. cornuta. e. Equatorial view. f. Polar view. g-h. S. haenkeana. g. Equatorial view. h. Polar view. i-j. S. heterophylla. i. Equatorial view. j. Polar view. k-1. S. lorentzii. k. Equatorial view. 1. Polar view. Scale bars $=5 \mu \mathrm{m}$.

molleoides (Vell) Engl., L. brasiliensis March, Schinus molle var areira (L.) DC., S. longifolius Speg. como tipo supraestriado-infrarreticulado.

La predominancia del carácter de la exina supraestriada reticulada, para la pared del polen de Schinopsis, muestra la importancia de la misma tanto a nivel de género como de familia; incluso su distribución en los granos de polen permite diferenciar a $S$. balansae del resto de las especies porque la misma es supraestriada-infrarreticulada en los mesocolpios y sólo estriada en áreas polares.

La forma de los granos de polen es otro carácter que permite agrupar algunas especies y delimitar otras. Así, $S$. haenkeana y $S$. lorentzii se diferencian palinológicamente, carácter morfológico que sustenta la propuesta de Mogni (2015) (en tabla 1, suboblato y prolato-esferoidal respectivamente) al determinar a ambos taxones como entidades diferentes a través de estudios moleculares.

\section{Conclusiones}

Este estudio permite concluir que considerando la distribución de la escultura de exina en los granos, la forma y el tamaño de los granos, y relacionándolos con el análisis de otros caracteres como es el tipo de hojas (v.g. en Mogni 2015), contribuyen 
a la delimitación taxonómica de las especies de Schinopsis aquí estudiadas, a pesar del carácter estenopalínico del género, así como lo indican otros análisis palinológicos realizados en varias especies de Anacardiaceae (Erdtman 1952, 1971, Anzótegui 1971, 2001, Ralph \& Robert 1979, Barros et al. 1999, Perveen \& Qaiser 2010). Esto se demuestra en la clave elaborada y presentada más abajo.
Además las nuevas descripciones polínicas de $S$. boqueronensis, $S$. cornuta y $S$. haenkeana (sensu stricto), permiten apoyar la propuesta de Anzótegui (2001) que propone como un único tipo polínico para la familia Anacardiaceae: tipo "Schinopsis balansae".

Algunos caracteres palinológicos y morfológicos foliares contribuyen a la delimitación de las especies estudiadas del género Schinopsis según la siguiente clave:

1. Hojas simples. Exina supraestriada-infrarreticulada en los mesocolpios, estriada en áreas polares ... 1.S. balansae

1. Hojas compuestas, o simples y compuestas en ejemplares adultos. Exina supraestriada-infrarreticulada en todo el grano.

2. Hojas compuestas pinnadas .Polen prolato esferoidal o suboblato

3. Polen de forma prolato esferoidal, con endoaperturas de $3,2 \times 3,6 \mu \mathrm{m}$ 6. S. lorentzii

3. Polen de forma suboblato, con endoaperturas de $2,5 \times 3 \mu \mathrm{m}$ 4. S. haenkeana

2. Hojas simples y compuestas pinnadas en ejemplares adultos

4. Polen de forma prolato 2. S. boqueronensis

4. Polen de forma suboblato, esferoidal o prolato esferoidal

5. Hojas usualmente con más de 5 folíolos. Polen suboblato a esferoidal con índice de área polar de $0,2 \mu \mathrm{m}$ 5.S. heterophylla

5. Hojas usualmente con 3 hasta 5 folíolos. Polen de forma prolato esferoidal con índice de área polar de $0,4 \mu \mathrm{m}$

3. S. cornuta

\section{Agradecimientos}

Agradecemos a las autoridades y curadores de los Herbarios (CTES, MCNS y UNR) que facilitaron las muestras para el estudio, a Silvia Blanco del Laboratorio de Microscopía Electrónica de Barrido y Microanálisis de la Universidad Nacional de Salta, por su ayuda con las imágenes de microscopía electrónica, y a los evaluadores anónimos por las valiosas sugerencias. Este trabajo fue financiado por Consejo Nacional de Investigaciones Científicas y Técnicas (CONICET), Consejo de Investigación de la Universidad Nacional de Salta (CIUNSa) y Universidad Nacional de Rosario (UNR).

\section{Literatura citada}

Anzótegui, L.M. 1971. Polen de Anacardiaceae del NE de Argentina. Ameghiniana 8: 329-340.

Anzótegui, L.M. 2001. Anacardiaceae.In: S.M. Pire, L.M. Anzótegui\& G.A. Cuadrado (eds.), Flora polínica del Nordeste Argentino 2: 19-26. EUDENE. UNNE.

Athavale Pradeep, S. 2014. LM and SEM Studies on the pollen morphology of family Anacardiaceae from Chandrapur and Gadchiroli districts of Maharashtra State. International Journal of Life Sciences: 11-12.

Barberis, I., Mogni, V.Y., Oakley, L., Alzugaray, C., Vesprini, J. \& Prado, D.E. 2012. Biología de especies australes: Schinopsis balansae Engl. (Anacardiaceae). Kurtziana 37: 59-86.
Barkley, F. 1962. Anacardiaceae: Rhoideae: Schinopsis. Proceedings of the Iraqi Scientific Societies 5: 44-69.

Barros, M.A., Barth, O.M. \& Costa, K.M. 1999. Catálogo sistemático dos pólens das plantas arbóreas do Brasil Meridional. XXXII: Anacardiaceae. Leandra 14: 17-24.

Cabrera, A. 1938. Revisión de Anacardiáceas austroamericanas. Revista del Museo de La Plata, Sección Botánica 2: 3-64.

del Pero de Martínez, M.A. 1972. Hibridación en el género Schinopsis. Resúmenes XII Jornadas Argentinas de Botánica, 2-5 Noviembre 1972, Neuquén, Argentina.

Erdtman, G. 1952. Pollen Morphology and Plant Taxonomy.Angiosperms.Chronica Botanica Co., Waltham, Mass. USA.

Erdtman, G. 1969. Handbook of Palynology: Morphology, taxonomy, ecology an introduction to the study of pollen grains and spores. New York: Hafner Publishing.

Erdtman, G. 1971. Pollen Morphology and Plant Taxonomy. Hafner Publishing Company, New York, USA.

Fernández, I. \& Diez, M. 1990. Algunas consideraciones sobre Terminología Palinológica, Polaridad y Simetría. Lagascalia 16: 51-60.

Flores, C.B., Zapater, M.A. \& Sühring, S. 2013. Identidad taxonómica de Schinopsis lorentzii y $S$. marginata (Anacardiaceae). Darwiniana, nueva serie 1: 25-38.

Judd, W., Campbell, C., Kellogg, E., Stevens, P. \& Donoghue, M. 1999. Plant Systematics: A Phylogenetic Approach. 2nd Edition. Sinauer Associates, Sunderland, Mass., USA. 
Markgraf, V. \& D'Antoni, H. 1978. Pollen Flora of Argentina. The University of Arizona Press. Tucson. AZ.

Meyer, T. \& Barkley, F. 1973. Revisión del género Schinopsis (Anacardiaceae). Lilloa 33: 207-257.

Mogni, V.Y. 2015. Estudio filogenético y biogeográfico del género Schinopsis (Anacardiaceae). Tesis Doctoral, Facultad de Ciencias Agrarias, Universidad Nacional de Rosario, Argentina.

Mogni, V.Y., Oakley, L., Vera Jiménez, M. \& Prado, D.E. 2014. A new tree species of Schinopsis (Anacardiaceae) from Paraguay and Bolivia. Phytotaxa 175: 141-147.

Mogni, V.Y., Prado, D.E. \& Oakley, L.J. 2017. Notas nomenclaturales en el género Schinopsis (Anacardiaceae). Boletín de la Sociedad Argentina de Botánica 52: 185-191.

Muñoz, J.D. 1990. Anacardiaceae. In: R. Spichiger\& L. Ramella (eds.). Flora del Paraguay 14: 7-84. Missouri Botanical Garden \&Conservatoire et Jardin Botaniques de la Ville de Geneve.

Pell, S. 2004. Molecular systematics of the cashew family (Anacardiaceae). PhD Dissertation, Louisiana State University, Baton Rouge, USA. Disponible en https:// digitalcommons.lsu.edu

Perveen, A. \& Qaiser, M. 2010. Pollen Flora of PakistanLXVI: Anacardiaceae. Pakistan Journal of Botany 42: 1401-1406.
Punt, W., Hoen, P.P., Blackmore, S., Nilsson S. \& Le Thomas, A. 2007. Glossary of pollen and spore terminology. Review of Palaeobotany and Palynology 143: 1-81.

Prado, D.E.1993a. What is the Chaco in South America? I. A review. Candollea 48: 145-172.

Prado, D.E.1993b. What is the Chaco in South America? II. A redefinition. Candollea 48: 615-629.

Prado, D.E. 2000. Seasonally dry forests of tropical South America: from forgotten ecosystems to a new phytogeographic unit. Edinburgh Journal of Botany57: 437-461.

Ragonese, A. \&Castiglioni, J. 1947. Nueva especie del género Schinopsis y área geográfica de las especies argentinas. Revista de Investigaciones Agrarias 1: 93-100.

Ralph, A. \& Robert, A. 1979. Pollen morphology of the Anacardiaceae of Northeastern North America. Bull. Torrey Botanical Club 106: 140-144.

Sáenz Laín, C. 2004. Glosario de Términos Palinológicos. Lazaroa 5: 93-112.

Thiers, B. 2015. Index Herbariorum: A global directory of public herbaria and associated staff. NewYork Botanical Garden's Virtual Herbarium. 